\title{
LA INFORMACIÓN CONTABLE EN LA ERA VIRTUAL Y DEL CONOCIMIENTO
}

CPC Miguel Diaz Inchicaqui*

\section{INTRODUCCIÓN}

La Contabilidad como la ciencia de la Información Empresarial a lo largo de su historia ha evolucionado buscando siempre satisfacer los requerimientos de los usuarios, sin embargo es notorio su estancamiento en las últimas décadas al no haber modificado la Información Financiera que reporta con relación a la evolución de la economía globalizada, la administración, la velocidad de los cambios tecnológicos y el incremento desmesurado del conocimiento.

Nuestra Investigación "La Información Contable en la Era Virtual y del Conocimiento", presenta los periodos que ha atravesado la contabilidad hasta llegar al siglo XXI, conocido como la Era del Conocimiento, en la cual las necesidades informativas han evolucionado de manera tal que nuestros usuarios solicitan información rápida y precisa a tiempo real, por el crecimiento incesante del comercio electrónico y la obligación de medir los activos intangibles para gestionar el conocimiento de los negocios; son los factores que impulsan nuestra investigación.

En este último período la contabilidad y la profesión contable ve cuestionada la Información Financiera que reporta a los usuarios, ya que estos estados financieros tradicionales que fueron diseñados para cumplir con los requerimientos de la era industrial, no exponen ni reflejan adecuadamente el valor real de las empresas, por lo que, este modelo tradicional que fue útil en su oportunidad muestra problemas fundamentales tales como:

- No mostrar el verdadero valor de las empresas que permita la toma de decisiones racionales de los usuarios. Actualmente estos problemas vienen siendo corregidos exclusivamente con nuevas reglas de valuación inmersas en las nuevas Normas Internacionales de Contabilidad.

- No identifica las fuentes de valor puestas a trabajar en una era virtual y de una economía del conocimiento.

Para cumplir con nuestro propósito, analizaremos las diferentes tecnologías de impacto que vienen afectando a la contabilidad, en un marco de comercio electrónico, el cual está obligando a nuestra profesión a revisar las prácticas y principios contables aún vigentes, con la finalidad de manejar adecuadamente la "Evidencia o Documentación Virtual", que será la generadora de la Información Financiera Virtual,

* Profesor Asociado de la Facultad de Ciencias Contables 
publicada en el internet $y$ probablemente en un futuro no muy lejano estaremos hablando de la Contabilidad y auditoría sin papeles de trabajo.

\section{MARCO DE LA EVOLUCIÓN HIS- TÓRICA DE LA CONTABILIDAD}

\section{LA EVOLUCión DE LA CONTABILIDAD}

Los profesionales en contabilidad estamos obligados en atender las necesidades de información de los usuarios que usan nuestros servicios, estas necesidades de información a través del tiempo ha venido evolucionando hasta llegar a nuestra actualidad el siglo XXI, donde prima la economia globalizada, el crecimiento incesante de las tecnologías de la información y la cultura del conocimiento.

Bajo este marco es interesante que nos preguntemos: ¿cuáles son los cambios fundamentales que han experimentado los sistemas empresariales desde que Luca Pacioli escribiera su obra? Lo que dijo Luca Pacioli, al comienzo del título noveno, tratado XI de la obra "De las Cuentas y las Escrituras" escrita en 1494 ..."Como es bien sabido, quien desee dedicarse al comercio y operar con la debida eficacia, necesita fundamentalmente tres cosas..."

- "La principal de ellas es el dinero..."

- "... la segunda cosa que se precisa para el tráfico mercantil es ser un buen contador y saber hacer las cuentas con gran rapidez..."

- "... la tercera y última cosa necesaria es la de registrar y anotar todos los negocios de manera ordenada, a fin de que se pueda tener noticia de cada uno de ellos con rapidez.....

Si analizamos lo que escribiera Pacioli en su tratado, podemos concluir que dos son los cambios fundamentales que se han producido en el comercio desde aquella época:

- En primer lugar los negocios son cada día más complejos, ya que en los tiempos de Pacioli no estaban desarrollados las fuentes de financiamiento tales como el leasing, leaseback, factoring o los mercados de opciones y futuros. Por lo que, esta evolución ha determinado que las necesidades de información que requieren los usuarios de la Contabilidad han cambiado.

- En segundo lugar observamos que han evolucionado las herramientas que posibilitan la labor del contable, tales como: la Estadística, la Investigación de Operaciones, etc. y más recientemente las tecnologías de la in formación y comunicación.

Donde Pacioli demanda "saber hacer las cuentas con gran rapidez", -hasta hace relativamente poco se exigía en las ofertas de empleo para contables "tener buena letra" - hoy exigiriamos "manejo fluido de programas de contabilidad", $y$ donde dice "anotar todos los negocios de manera ordenada" hoy añadiriamos "conocimientos de informática a nivel del usuario». 


\section{ETAPAS DE LA CONTABILIDAD}

La Contabilidad a través del tiempo ha atravesado las siguientes etapas históricas:

\begin{tabular}{|c|c|c|c|}
\hline $\begin{array}{l}\text { PERIODO } \\
\text { HISTÓRICO }\end{array}$ & $\begin{array}{l}\text { NECESIDADES } \\
\text { INFORMATIVAS }\end{array}$ & $\begin{array}{l}\text { POSIBILIDADES } \\
\text { TECNOLÓGICAS }\end{array}$ & $\begin{array}{l}\text { RESPUESTA DE LA } \\
\text { CONTABILIDAD }\end{array}$ \\
\hline $\begin{array}{l}\text { Las Grandes } \\
\text { Civilizaciones }\end{array}$ & Conocer los ingresos y gastos & $\begin{array}{l}\text { Papiro, escritura } \\
\text { cuneiforme }\end{array}$ & Utilizar la partida simple \\
\hline $\begin{array}{l}\text { El inicio del } \\
\text { comercio }\end{array}$ & Registrar cada movimiento & Papel & $\begin{array}{l}\text { Surge la partida doble. Pri- } \\
\text { meros libros contables }\end{array}$ \\
\hline $\begin{array}{l}\text { La Revolución } \\
\text { Industrial }\end{array}$ & $\begin{array}{l}\text { Importancia de los activos y } \\
\text { conocer el beneficio }\end{array}$ & Papel, imprenta & $\begin{array}{l}\text { Se perfecciona la par- } \\
\text { tida doble. Estados fi- } \\
\text { nancieros. }\end{array}$ \\
\hline 1960 & $\begin{array}{l}\text { Manejar más información y } \\
\text { con más rapidez }\end{array}$ & $\begin{array}{l}\text { Los primeros ordenado- } \\
\text { res: muchos usuarios } \\
\text { para un equipo }\end{array}$ & $\begin{array}{l}\text { Se automatizan los sis- } \\
\text { temas contables ma- } \\
\text { nuales. }\end{array}$ \\
\hline 1981 & $\begin{array}{l}\text { Obtener información finan- } \\
\text { ciera útil para la toma de } \\
\text { decisiones }\end{array}$ & $\begin{array}{l}\text { Ordenador personal: la } \\
\text { informática se populariza }\end{array}$ & $\begin{array}{l}\text { Sistemas de información } \\
\text { contables integrados en } \\
\text { bases de datos. Infor- } \\
\text { mes, ratios, gráficos. }\end{array}$ \\
\hline Siglo XXI & $\begin{array}{l}\text { - Información en tiempo real. } \\
\text { - Comercio electrónico. } \\
\text { - Medir activos intangibles } \\
\text { para gestionar el conocimiento. }\end{array}$ & $\begin{array}{l}\text { - Ordenadores en red: } \\
\text { Internet } \\
\text { - Tecnologías de la } \\
\text { cormunicación }\end{array}$ & $\begin{array}{l}\text { - Automatizar } \\
\text { captura de datos. } \\
\text { - Intercambio electró- } \\
\text { nico de documentos. } \\
\text { - Desaparece el papel }\end{array}$ \\
\hline
\end{tabular}

Tabla: Etapas de la Evolución de la Contabilidad

\section{LA REVOLUCIÓN DE LAS TELECOMUNICACIONES}

Es notorio el efecto de la revolución de las telecomunicaciones en la contabilidad ya que desde el punto de vista de la tecnología nos encontramos en la tercera revolución informática, la de las comunicaciones, caracterizada por un ratio de una persona con muchos ordenadores. La utilización intensiva de herramientas ligadas al sector de las telecomunicaciones introducirá cambios importantes en muchas de las actividades típicas del contador. Por ejemplo, Internet, las aplicaciones de Internet como el World Wide Web, el correo electrónico, las listas de distribución, los boletines de noticias, el acceso remoto a cualquier ordenador de Internet, los sistemas para transferir ficheros, la utilización de agentes informáticos para realizar informes que combinen información interna y externa, las charlas interactivas y las videoconferencias son algunas de las tecnologias con gran impacto en las actividades típicas del contador. 


\section{COMPARTIR EL CONOCIMIENTO}

El Contador Público en el siglo XXI dispone de nuevas herramientas informáticas que tratan de administrar, compartir y canalizar tanto la información real como el conocimiento intangible de la empresa de forma que éste fluya en la organización integrado en aplicaciones como las Intranet, Groupware, Data Mining, Knowledge Data Base, gestión de documentos, etc., aunque de entre todas ellas destaca el Intercambio Electrónico de Datos o EDI. Cuando el EDI está perfectamente integrado con el resto de aplicaciones informáticas, la mayoria de los asientos contables se realizan de forma automática. Actividades como la facturación o el control de las existencias, con el EDI son automáticas. Será entonces el EDI el que conduzca al fin de la imagen del Contador Público rodeado de papeles, por pedidos, control de stocks y facturas.

\section{LA CONTABILIDAD EN LA ERA DEL CONOCIMIENTO}

En la era del conocimiento el profesional contable tiene que tomar en cuenta los siguientes conceptos:

\section{LAS ORGANIZACIONES BASA- DAS EN LA INFORMACIÓN}

La empresa como sistema que recibe unos input, que son los recursos que se procesan en sus diferentes departamentos, y a través de la venta de ellos obtendrán un output, que a su vez son un input para otras empresas. Como observamos, lo que procesa el sistema son los recursos básicos tradicionales como: La Materia prima, Máquinas, el dinero que es el capital y los recursos humanos. Sin embargo, en la Era del Conocimiento, podemos considerar a la información como un recurso fundamental que también ha de añadirse a la lista anterior. Además, está surgiendo un nuevo tipo de empresa, que se suelen denominar: "organizaciones basadas en la información". Para Drucker (1993) uno de los rasgos que caracterizan a las organizaciones basadas en la información es la reducción del número de niveles jerárquicos hasta la mitad. Sobre todo son los mandos intermedios, los que están llamados a disminuir, ya que muchas veces realizan un mero papel de intermediarios entre los empleados y la dirección, elaborando información obtenida del control a los trabajadores, para que los directivos puedan tomar decisiones.

\section{LOS SISTEMAS DE INFOR- MACIÓN}

Un sistema de información es un conjunto de elementos interrelacionados que recoge datos, los procesa y convierte en información, que almacena y posteriormente distribuye a sus usuarios. Como todo sistema, incluye también un ciclo de retroalimentación que es un mecanismo de control. En toda empresa conviven varios sistemas o mejor dicho, subsistemas- de información, como el de márketing o producción. 


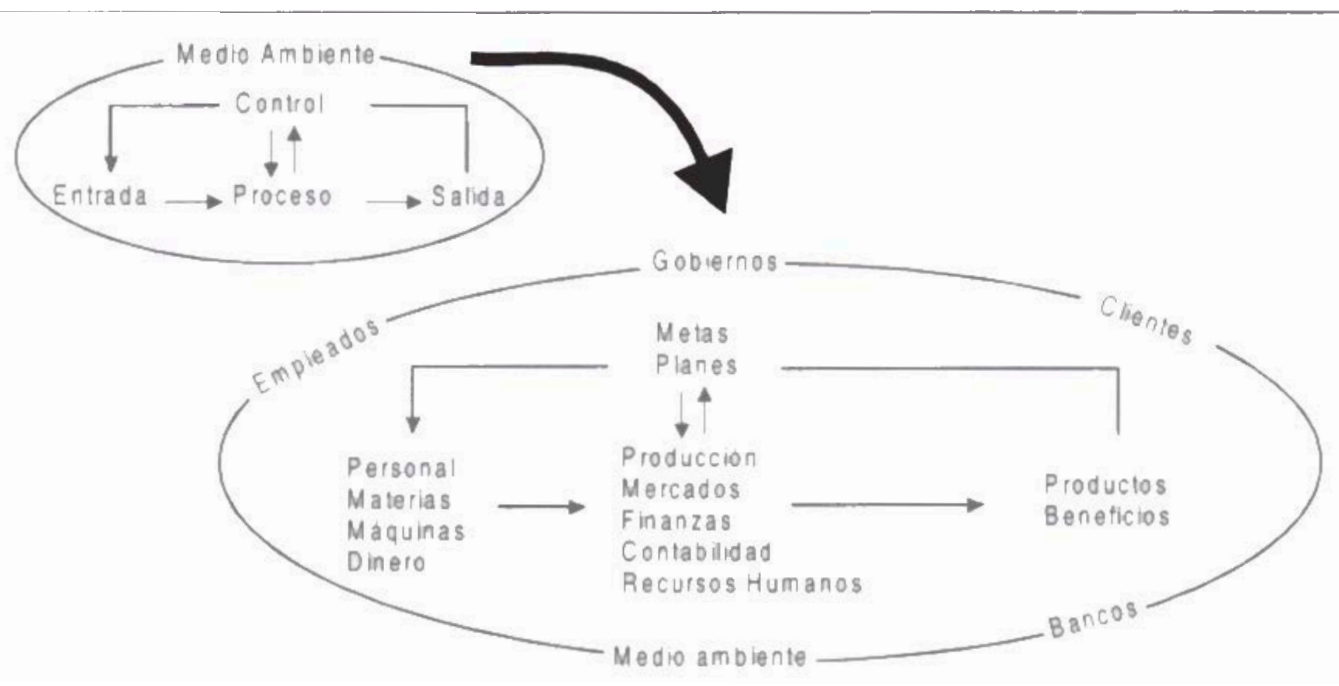

Figura: La empresa como sistema de información

\section{LOS SISTEMAS DE INFOR- MACIÓN CONTABLE}

La Contabilidad es un sistema de información ya que captura, procesa, almacena y distribuye un tipo particular de dato, la información financiera, vital para la correcta toma de decisiones en la empresa. Aunque en las empresas hay sistemas de información que son informales y no están informatizados - como las redes de circulación de rumores en una oficina-, el sistema informativo contable actual se basa en los ordenadores como pieza clave del mismo. La Contabilidad como sistema de información se ha adaptado a las necesidades informativas de sus usuarios, pero su desarrollo ha estado limitado por los recursos tecnológicos. Es decir, cuando el Contador Público se plantea una primera pregunta: en esta empresa y momento del tiempo ¿qué información demandan los usuarios? Un rasgo que hace que el sistema de información contable sea peculiar es la existencia de usuarios internos - como el gerente- y externos - como las autoridades tributarias- que demandan información contable. Con la tecnología actual, ¿qué información puedo darles? Hace varios años aunque los directivos de una empresa demandaran un balance mensual, era muy costoso obtenerlo con una contabilidad manual, hoy en día cualquier programa le permite hacer cierres virtuales de la contabilidad pulsando una tecla. Como vemos, es muy importante para el contador conocer el estado de la tecnología de la información en cada momento.

\section{LAS NECESIDADES DE INFOR- MACIÓN}

Las nuevas necesidades informativas en la Era del Conocimiento, las tecnologias actuales y los cambios que experimenta la Contabilidad, deben dar respuesta a la demanda de 
información a través del uso de las tecnologías que más impacto puede tener en la profesión contable

La Contabilidad ha evolucionado según las necesidades de información que demandan sus usuarios, de necesidades de información tradicionales hoy en dia en plena Era del Conocimiento surgen nuevas necesidades de información, tales como: Identificar y valorar el capital intelectual de las empresas, para desarrollar la planificación, control, información y auditoría; para esto la contabilidad del capital intelectual requerirá la invención de nuevos conceptos y prácticas contables.

Para cubrir estas nuevas necesidades que requieren los usuarios es necesario modificar los sistemas de información vigentes, es decir, información del nivel estratégico, que no están cubiertas por los estados contables tradicionales tales como:

- información sobre los riesgos a que está sometida la empresa,

- los recursos humanos,

los activos intelectuales,

- el impacto medioambiental,

Las impresiones de los clientes sobre la calidad de nuestros productos, y un largo etcétera.

Estas son nuevas necesidades de información que tienen los gerentes de las empresas y los analistas externos, generadas en la llamada Era del Conocimiento.

Por ejemplo: valorar el capital intelectual

Uno de los recientes debates sobre a dónde debe ir la Contabilidad, trata sobre si debe incluirse el llamado capital intelectual en los estados financieros de las empresas. Esta postura enlaza con las recientes teorías de economía de empresa ligadas a la gestión del conocimiento en la empresa y al éxito que están teniendo empresas que destacan más por sus activos intangibles como la imaginación, capacidad de inventiva, grado de satisfacción de los clientes o la motivación de los empleados, etc. que por su activo fijo o su estructura financiera. Es decir, se trata de valorar los recursos intelectuales de las empresas, como un activo intangible más, y crear nuevos documentos contables. Es también un área de interés en otros campos como la Sociologia por cuanto involucra gestión de recursos humanos.

Otros usuarios de la información contable -como los analistas externos usuarios de la información financiera que quieren utilizar la información para la tarea de la toma de decisiones y no tienen acceso al sistema informativo de la firmatambién manifiestan su decepción ante la información contable suministrada por las empresas y su interés en conocer más sobre los activos intangibles de las mismas.

Además, estos profesionales demandan sobre todo que la información contable sea oportuna en el tiempo. Hoy en día información oportuna es información en tiempo real.

Sirva de ejemplo extremo el caso de empresas que operan en Internet como Terra, Excite, Yahoo!, Netscape, Amazon, Cybercash, Geocities y tantas otras empresas con estados financieros que sistemáticamente presentan 
pérdidas, pero cuyos activos intangibles - por haber posicionado unas marcas, o conocer sus directivos y empleados los entresijos del comercio electrónico, o haber desarrollado unos sistemas de información o canales de distribución eficientes, etc. - tienen un valor real impresionante.

Como vemos, el valor de los activos intangibles distorsiona la mayoría de los indicadores del análisis contable, para ello debemos de tomar en cuenta la posibilidad de registrarlo como un activo fijo estimando su vida útil y determinando su valor objetivo, para exponerlo en los estados financieros.

\section{EL IMPACTO DE LAS TECNOLOGĹAS EN LA CONTABILIDAD}

El impacto de la tecnologia en la profesión contable está fuera de toda duda. Las tecnologías de la información operan como motor del cambio que permite dar respuesta a las nuevas necesidades de información. Para Elliot (1992) las tecnologías de la información son el motor del cambio que conduce a una nueva era postindustrial que amenaza con dejar obsoletas todas las estructuras empresariales que no sepan adaptarse.

En cuanto al impacto de las tecnologías de la información en las prácticas y sistemas de información contables es necesario distinguir entre aquellas mejoras que sólo afectan a aspectos cuantitativos y aquellas que permiten que nuevos métodos de trabajo contable puedan llevarse a cabo o permiten diseñar nuevos sistemas de información. Entre los primeros podemos citar la mayor velocidad que supone utilizar un soporte informático para obtener los balances o la posibilidad de depositar las cuentas en los registros mercantiles o liquidar los impuestos a través de procedimientos de intercambio electrónico de información. Esto es muy positivo por el ahorro en tiempo y dinero que supone pero no introduce cambios metodológicos.

Un ejemplo, Microsoft: el "sistema nervioso digital" y la Contabilidad "En un articulo publicado por Scott M. Boggs, controller de Microsoft, en mayo de 1999 en el Journal of Accountancy, comentan que hace cinco años, a Microsoft le costaba dos semanas de cada mes cerrar sus libros y ahora cuatro días. El objetivo previsto es lograr un cierre de libros continuo y diario. 350.000 copias en papel de informes de gestión han sido reemplazadas por documentos online. Hoy no generan ni una hoja de papel. Ninguna de la tecnología que ellos usan esta fuera del alcance de una empresa, grande o pequeña. Utilizan una intranet, llamada Microsoft FinWeb (Financial Information Network) un data warehouse o almacén de datos que extrae información del diario y la pone accesible en una base de datos de Microsoft Access. Otras herramientas son tablas dinámicas de Excel (PivotTable) que permite profundizar en los detalles de cada división, producto, mercado, etc. que se complementa con consultas SQL mediante una herramienta llamada MS Report.

Otra herramienta interesante es MAP (Microsoft Accounting Policies), una parte de la intranet que archiva las politicas contables de la empresa, sobre 500 aspectos (por ejemplo, cómo contabilizar los gastos de automóvil." 
Cisco Systems el fabricante líder en Internet de componentes, redes, etc., cierra sus libros de Contabilidad en il dia!, y publica los resultados en Internet seis días después.

\section{LAS TECNOLOGIAS DE LA INFOR- MACIÓN GUE DEBE CONOCER EL CONTADOR PÚBLICO}

En esta sección veremos lo que recomiendan diversas asociaciones internacionales de contables a los profesionales y estudiantes de Contabilidad.

\section{LO GUE RECOMIENDA LA FEDERACIÓN INTERNACIONAL DE CONTADORES-IFAC}

\subsection{Los Contadores Públicos debemos} saber cuatro cosas, de acuerdo con la Guía Internacional de Educación $\mathrm{N}^{\circ}$ 9, «Educación de Precalificación, Valoración de la Competencia Profesional y Requerimientos de Experiencia de Contables Profesionales", de IFAC, la Federación Internacional de Contables - el conocimiento que los aspirantes a trabajar como contables deben adquirir se asienta en cuatro componentes:

a) Conocimiento en Contabilidad -que proporciona el trasfondo técnico esencial

b) Conocimiento general -que cubre un amplio rango de temas en las artes, ciencias y las humanidades

c) Conocimiento organizacional y de negocios -que es el contexto en el que trabajan los contadores.

d) Conocimiento en tecnologia de la información
1.2 Tecnología de la Información, en cuanto al conocimiento en tecnología de la información, es desarrollada en la Guía Internacional de Educación $\mathrm{N}^{\circ}$ 11, "Tecnología de la Información en el curriculum de Contabilidad" revisada en junio de 1998. Para IFAC, el profesional de la Contabilidad no sólo tiene que usar los sistemas de información, sino que también desempeñar un papel importante en el diseño, administración y evaluación de tales sistemas. Por lo tanto, los Contadores Públicos, tanto los que trabajan en la industria o comercio, práctica profesional o el sector público adoptan diferentes papeles frente a las tecnologías de la información, que para IFAC son cuatro:

a. Usuario de tecnologías de la información, para IFAC, durante la etapa de aprendizaje previa al mundo laboral todos los futuros profesionales de la contabilidad deben obtener un conocimiento general de tecnologías de la información $y$ habilidades prácticas y el conocimiento correspondiente al nivel de usuario.

b. Administrador de sistemas de información, el profesional de la contabilidad puede tener responsabilidades compartidas sobre la administración de sistemas de información de una pequeña y mediana empresa. Los contenidos teóricos que debe conocer el contable que se identifique con este papel son relativos a las consideraciones estratégicas del desarrollo de tecnologias de información, aspectos administra- 
tivos, control financiero de las tecnologias, aspectos operativos, de seguridad, backup y recuperación, gestión de adquisición, desarrollo e imple-mentación de sistemas, gestión de cambio y mantenimiento de sistemas y gestión de computación de usuario final.

Diseñador de sistemas de negocios, en el caso del diseñador de sistemas, los profesionales de la contabilidad desde siempre han estado involucrados en el diseño de sistemas de información contables, antes cuando eran manuales, pero hoy también se espera que continúen proporcionado dichos servicios, como parte de un equipo multidisciplinar. Los conocimientos que se espera que tenga están relacionados con el papel de la información en el comportamiento y diseño de la organización, análisis de sistemas, las fases, tareas y prácticas de desarrollo del ciclo de vida de los sistemas de información, en particular sobre los controles necesarios.

- Evaluador de sistemas de información, el papel del Contador Público como evaluador de sistemas está relacionado con la auditoría tanto interna como externa. Los conocimientos que debe poseer se relacionan con los aspectos normativos referidos a legalidad, ética, auditoria y control relativos a las tecnologías de la información. En concreto se refieren a la evaluación de la eficacia, eficiencia y economicidad del uso de las tecnologias de la información, evaluación de su conformidad con política de gestión, evaluación de los controles internos en sistemas informatizados y evaluación de la imparcialidad de la imagen financiera, la veracidad e integridad de los registros contables.

\subsection{Tendencias en Tecnologias de} Información (IFAC). Las tecnologías de la información están cambiando la forma en que los contadores trabajan y el marco en el que se desarrollan sus actividades, porque han cambiando la forma en que las empresas se organizan y gestionan. De acuerdo con el IFAC, son particularmente dignas de mención las siguientes tendencias en Tecnologias de la Información (TI):

a) La amplia disponibilidad de potentes pero económicos equipos informáticos.

b) La amplia disponibilidad de programas de ordenador potentes, económicos y relativamente sencillos de usar con interfaces de usuario gráficas.

c) El paso de adquirir sistemas informáticos a medida a utilizar software "llave en mano".

d) $\mathrm{El}$ paso de grandes equipos centralizados o "mainframes" a ordenadores personales, utilizados solos o como parte de redes.

e) La disponibilidad creciente de datos informatizados a los que se accede en tiempo real o diferido, mediante acceso local o remoto, incluido via Internet.

f) Las nuevas tecnologías de captura de datos y almacenamiento llevan a la informatización de datos e información en formato de texto, gráficos, audio y video y enfatizan el gestionar, presentar y comunicar información utilizando técnicas multimedia. 
g) La convergencia de las tecnologías de la información y la comunicación, que afecta a cómo las personas trabajan y compran.

h) El uso creciente de redes que conectan a los individuos y a las empresas -intraempresas e interempresas - con sistemas como el correo electrónico e Internet, incluyendo el World Wide Web.

i) El uso creciente de Internet para el comercio entre empresas y la venta de productos finales a un consumidor, vía sistemas de comercio electrónico como el Intercambio Electrónico de Datos (EDI) y sistemas de transferencia electrónica de fondos (EFTS).

j) El marketing y la distribución masiva de productos de tecnologias de la información y servicios tales como ordenadores, programas informáticos "llave en mano", servicios de recuperación de datos on-line, correo electrónico y servicios financieros.

k) La reducción de barreras al uso de sistemas, la prometedora y más amplia incorporación de sistemas de información en organizaciones lucrativas y no lucrativas de todo tipo para temas de contabilidad, de gestión y estratégicas y el uso creciente de la informática de usuario final.

1) Amplia penetración de tecnologias de la información como diseño y manufactura asistidos por ordenador (CAD/CAM), sistemas de información para la dirección (EIS o executive information systems) y sistemas de reunión electrónica (EMS o electronic meeting systems).

m) Las nuevas técnicas de desarrollo de sistemas basadas en tecnologias de información como las herramientas de ingeniería del software (CASE u computer-assisted software engineering), programación orientada a objetos y tecnologias work-flow.

n) Desarrollo continuado de sistemas de ayuda inteligentes que incorporan sistemas expertos, redes neuronales, agentes inteligentes y otras técnicas de resolución de problemas.

o) Nuevas metodologías de reingenieria de negocios basadas en la integración efectiva de tecnologias de información y procesos de negocio.

\section{LAS TECNOLOGIAS MÁs RELE- VANTES PARA THE AMERICAN INSTITUTE OF CERTIFIED PUB- LIC ACCOUNTANTS (AICPA)}

Otras instituciones también recomiendan que los Contadores Públicos dominen ciertas tecnologias. Por ejemplo, The American Institute of Certified Public Accountants (AICPA) realiza un congreso anualmente al que asisten Contadores Públicos expertos en tecnologias y votan cuales son las tecnologias de mayor impacto para la profesión contable.

También podemos leer en la revista Journal of Accountancy que edita AICPA los resúmenes anuales sobre tecnologías de impacto.

En CPAvision, servidor de AICPA $y$ VisonNet, podemos encontrar varios documentos sobre el futuro de la Contabilidad.

\subsection{En Cuanto a Tecnologías E1} AICPA. Se refiere a las 10 innovaciones en hardware o soft- 
ware que más impacto puede tener en la Contabilidad, tales como:

Tecnologías relativas a la seguridad de los sistemas de información, actividades relacionadas con las copias de seguridad, encriptar un fichero u obtener una firma digital, es decir saber enfrentar los riesgos y amenazas de errores humanos, fallas en los equipos, robo, virus, sabotaje, fraude, etc.

- XML (Extensible Markup Language), para hacer páginas web se utiliza el lenguaje HTML, muy útil para las presentaciones, formatos, colores, etc. El HTML ha logrado que Internet se convierta en una enorme colección de documentos. Pero para desarrollar el comercio electrónico, intercambio de documentos mercantiles, cuentas anuales, se queda corto. Es más apropiado su hermano XML, que puede lograr que Internet se convierta en una gigantesca base de datos. Pensemos en el impacto de esta tecnologia en temas que afectan a la Contabilidad, como: el procesamiento de pedidos, facturas, estandarización de la información contable, etc.

- Tecnologías de la comunicación. Ancho de banda, es la máxima cantidad de datos que pueden pasar por un medio de comunicación en un momento dado, medido en segundos. Numerosas aplicaciones requieren gran ancho de banda: Uso de Redes en Educación a Distancia, Transmisión de video y Conferencias, Gestión remota, Almacenamiento Distribuido de los datos, Manejo de Imágenes, herramien- tas de trabajo en grupo, Realidad Virtual, etc.

Tecnologías móviles, como los teléfonos móviles con WAP o UMTS, ordenadores portátiles, ordenadores que caben en la palma de la mano están llamados a ser una de las tecnologías clave en el futuro próximo, con muchas aplicaciones para que los auditores, asesores, contables puedan trabajar o dar servicio a los clientes. Favorecen la oficina móvil, permiten acceso a Internet e Intranets, a bases de datos corporativas, videoconferencia, transferencia de ficheros, etc.

Wireless Technologies (includes wireless networks), esta tecnología trata de facilitar la comunicación entre dispositivos sin la utilización de cables aprovechando la movilidad de los dispositivos inalámbricos.

Verificación electrónica, uno de los aspectos claves del comercio electrónico: ¿Cómo sé yo que tú eres quien dice ser?, incluso en un programa de correo electrónico es sencillo suplantar la personalidad de otra persona simplemente cambiando la configuración. Muchas tecnologias pretender resolver esta cuestión: desde el uso de passwords, reconocimiento de voz o entidades de certificación, que permiten la verificación de identidad de una persona o entidad que quiere utilizar la firma electrónica, o la autentificación de servidores de comercio electrónico.

Encriptación, es un proceso por medio del cual se transforma un mensaje usando una clave o algoritmo es decir se traduce los signos de un mensaje a otro siste- 
ma de signos cuya lectura no tenga ningún sentido para un desconocido que los intercepte.

- Autorización electrónica, son soluciones técnicas que permiten que muchas tareas se aprueben electrónicamente, desde contratos a órdenes de compra.

- Herramientas de conectividad remota, son tecnologias que permiten el acceso y control remoto de ordenadores. Un profesional puede acceder a un ordenador remoto desde su despacho o desde su casa y ejecutar un programa de gestión, contabilidad, etc.

- Bases de Datos, tecnología usada para mantener las bases de datos con información relevante $\mathrm{y}$ actualizada.

2.2 En Cuanto a Aplicaciones El AICPA. Se refiere a las 10 aplicaciones, entendidas como oportunidades de negocio que utilizan una o varias tecnologias que más impacto puede tener en la Contabilidad.

- Aplicaciones basadas en el Web (Web-Based), Internet, y en concreto el WWW es una de las invenciones más importantes, con gran impacto en la vida de las personas y de los contables que pueden hacer un pedido en cualquier parte del mundo, pagarlo y recibirlo por correo electrónico. Es tan importante que combina o integra casi todas las tecnologías de la lista.

- Gestión del conocimiento, los sistemas para gestionar el conocimiento incluyen aplicaciones de programas diseñadas para opti- mizar el trabajo en grupos. Abordan desde las videoconferencias a través de Internet, las audio conferencias, la compartición de pantallas o los programas de charlas. Pero también otras herramientas que permiten gestionar agendas, organizar reuniones, organizar documentos y presentaciones en pantalla.

- Transmisión de información financiera, los nuevos estándares de Internet, como el citado XML permitirán preparar, publicar intercambiar y analizar informes financieros en una variedad de formatos. Nuevas posibilidades surgen para los Contadores Públicos.

- Aplicaciones Web-Enabled, sin llegar a ser aplicaciones "basadas en web", que sería un paso más, es relativamente sencillo modificar programas y dejarlos listos para ser utilizados vía Internet con el objetivo de obtener más prestaciones.

- Detección de intrusos, desde la Moncloa hasta Microsoft son muchas las entidades que han visto como sus ordenadores sufrían ataques procedentes de intrusos. Los intrusos entran a un sistema normalmente como invitados y tratan de explotar algún defecto del sistema para llegar a controlarlo.

- Data Mining, consiste en explorar automáticamente grandes bases de datos para extraer patrones de comportamiento, relaciones ocultas entre las variables, identificar dependencias. El data mining nos ayuda a encontrar qué clientes tienen más probabilidad de responder al próximo mailing promocional, descubrir 
patrones de comportamiento en consumidores o encontrar cuales son los mejores y peores clientes de una compañía de seguros o un banco.

- Gestión de documentos, la mayor parte de las empresas siguen imprimiendo casi todos los documentos que generan. Para la empresa supone un coste muy elevado crear, gestionar y distribuir documentos. Estas herramientas permiten publicar, distribuir $y$ gestionar grandes cantidades de información en la empresa utilizando varias tecnologias. El objetivo de estas aplicaciones es que los empleados de la organización puedan encontrar la información que necesitan tanto en bases de datos como en correos electrónicos, faxes, cartas, documentos, recortes de prensa, etc.

- Auditoría continua, ¿Auditoría en tiempo real? Quizá sea lejana pero lo cierto es que la auditoría clásica de los estados financieros está perdiendo importancia ya que los usuarios reclaman información en tiempo real. En el nuevo marco que supone la publicación de información online con una menor periodicidad surge la necesidad de desarrollar un modelo de auditoria continua.

- Aprovisionamiento electrónico, e-procurement, tiene como objetivo optimizar los procesos de aprovisionamiento de las empresas mediante la creación de centros virtuales de compras personalizados para cada empresa, utilizando las ventajas que Internet ofrece en este tipo de transacciones. Las empresas pueden realizar todas sus compras (ma- terial de oficina, equipos informáticos, piezas de recambio, servicios de impresión, viajes de empresa, etc.) de forma electrónica.

- Depósito electrónico de documentos contables o fiscales, la posibilidad de depositar las cuentas anuales en registros mercantiles o liquidar los impuestos vía Internet.

\subsection{En Cuanto a Situaciones de} Negocio El AICPA. Se refiere a las 10 situaciones de negocio que resultan una vez que las aplicaciones o tecnologias se han implantado.

- Aspectos de seguridad y control de la información, mantener la información segura en la Era de la Información se ha convertido en una prioridad. Por lo que, mantener un plan de seguridad de la información de una organización debe tratar todas las amenazas internas o externas a la organización.

- Comercio electrónico, es el nuevo marco de negocios en el que se desarrollan cada vez más operaciones mercantiles. En el comercio electrónico convergen tecnologias que ya existian aisladamente tan dispares como el Intercambio Electrónico de $\mathrm{Da}$ tos, el tele trabajo, la publicidad en Internet, las Intranet o las compras electrónicas, pero que tienen en común el uso de las telecomunicaciones.

- La nueva economía y la información financiera, si el modelo contable actual es bastante criticado por poco útil, en las nuevas empresas que utilizan Internet como parte central de sus ne- 
gocios es mucho más, porque en una nueva economia estas empresas que asumen nuevos riesgos, que tienen en sus activos intangibles un valor muy importante que no se refleja en la contabilidad, nacen crecen y mueren a una velocidad increible aspecto que aún no puede ser registrado por la contabilidad. La contabilidad en la Era del Conocimiento debe adaptarse a las necesidades de los usuarios y este tipo de empresas es un ejemplo extremo.

Privacidad, es un requisito tanto para los consumidores como para las empresas, que se ven afectado por el comercio electrónico. Las empresas tienen la responsabilidad de proteger los datos de los clientes.

Aprendizaje y tecnologia, la tecnología está cambiando las formas en que se captura, procesa y distribuye la información financiera. Por lo que, uno de los aspectos más importantes para el contador es la parte formativa del uso de las mismas con la finalidad de sacar el mayor provecho a dichas tecnologias.

Recuperación tras el desastre, disponer de un buen plan de recuperación de desastre ante posibles eventualidades, hará un negocio más seguro. Podemos decir con ironia que las empresas están perfectamente preparadas para afrontar con éxito la segunda vez que sufren un desastre.

Personal calificado en Tecnología de Información, al calor de esta tercera revolución de la informática, el personal especializado en tecnologias de la información es muy demandado. Las ofertas de empleo se canalizan cada vez más via Internet, lo que se denomina E-recruiment.

Calidad de servicio, cualquier problema en los sistemas de información repercute instan-táneamente en la empresa y afecta su funcionamiento normal. Es decir, tareas como por ejemplo la contabilidad financiera depende de lo que pasa en el ordenador. Cuando una empresa decide instalar o poseer una infraestructura informática, la gestión de tales recursos debe buscar el cumplimiento de tres objetivos.

Disponer del nivel de servicio más apropiado para que los usuarios desempeñen adecuadamente sus responsabilidades.

Reducir al máximo los riesgos derivados de la falta de seguridad y del uso inadecuado o fraudulento de la información.

Lograr una utilización eficiente de los recursos asignados a dicho centro de cálculo mediante las oportunas medidas de control.

- Auditoria en entornos de trabajo electrónicos, al desaparecer el papel, los contadores han familiarizarse y confiar en la evidencia electrónica para muchos propósitos. Algunos de los métodos que están disponibles validar y para autentificar evidencia incluyen firmas digitales, filigranas (watermarks) y certificados.

2.4Tecnología Emergentes. Se refiere a las 5 innovaciones en hardware o software más futuristas que no hay que perder de vista, entre ellas tenemos: 
- Regulaciones gubernamentales que tratan sobre tecnologia, en el Perú también se ha implementado normas reglamentarias.

- Business Service Providers (BSP), el Mercado de servicios de los proveedores son variados y de fácil accesibilidad por los usuarios.

- E-Learning, o metodologias didácticas.

- Evidencia Electrónica, imaginemos que el programa que gestiona las existencias detecta que falta un material y automáticamente hace un pedido al proveedor, quien también automáticamente da la orden de servir los productos a nuestro almacén y envía una factura electrónica así como la orden de pago vía banca electrónica. Todo el mundo está emocionado por la eficacia y ahorro de costes... excepto los auditores, que, además, están poco acostumbrados a trabajar "sin papeles".

- M-Commerce (comercio móvil), podemos definir el $\mathrm{M}$-commerce como el uso de la tecnología de comunicaciones móviles para comunicar, formar, informar, realizar transacciones y operar en las actividades personales y de negocios.

\section{LAS NUEVAS CARACTERÍSTICAS $Y$ PRÁCTICAS CONTABLES}

\section{LOS EFECTOS EN LOS PROCE- SOS CONTABLES}

1.1 La captura de datos debe simplificarse. En cuanto al proceso de identificar y registrar los hechos económicos, el primer paso es la captura de datos en el or- denador, actividad que realiza el administrativo contable. El ordenador facilita la labor del contable a la hora de registrar las operaciones habituales dentro de la organización: compras, ventas, gastos, amortizaciones, etc. Legiones de administrativos. Aún así, la captura del dato contable es una de las actividades que mảs esfuerzo administrativo precisa, con legiones de administrativos dedicados a, antiguamente llevar los libros, y, hoy en dia, introducir datos en un ordenador. Nuevas posibilidades Aunque el impacto de los ordenadores ha sido muy notable, facilitando este trabajo, en muchos sectores esta actividad puede conocer nuevas posibilidades con la captura automática de información, con mecanismos como el escáner y los códigos de barras, el control por la voz, los sistemas de transmisión por radiofrecuencia, etc. En el marco del Comercio Electrónico, esta actividad administrativa puede desaparecer.

\subsection{La captura de datos debe au-} tomatizarse. Con Intercambio Electrónico de Datos (EDI), Consiste en transmitir electrónicamente documentos contables entre aplicaciones informáticas en un formato normalizado. El EDI es una forma de Comercio electrónico de Empresa a Empresa (B2B). Si la empresa dispone de algún sistema de Intercambio Electrónico de Datos o EDI la captura de datos puede mecanizarse en buena parte, ya que los datos se introducen una única vez en el sistema de información del cliente al realizar un pedido y de ahi pasan automáticamente al sistema de información del pro- 
veedor. ¿Cómo se simplifica la captura de datos con EDI? Las empresas que hacen EDI simplifican la captura de datos. Para hacernos una idea es como un programa de correo electrónico sólo que estamos enviando pedidos, facturas, etc. en un formato normalizado, que "entienden" los ordenadores. No hace falta volver a meter los datos dos veces.

\subsection{El procesamiento de los datos} debe automatizarse al máximo. El sistema de información contable desempeña un papel fundamental en la medición de los resultados financieros. En cuanto al procesamiento de los datos contables, hoy en dia está bastante automatizado en la mayor parte de las empresas. Huelga repetir el impacto que la informática ha supuesto en todos los aspectos de gestión de la contabilidad: diario, mayor, cuentas a cobrar y pagar, elaboración de la nómina, obtención estados contables como el balance, la cuenta de pérdidas y ganancias, el cuadro de financiación o el estado de valor añadido proporcionan información contable cuantitativa. Debido a que la información cualitativa es más difícil de tratar con las tecnologías actuales, documentos como la memoria no están completamente automatizados.

\section{LA OFICINA SIN PAPELES, UNA TENDENCIA gUE CRECE EN AMÉRICA LATINA}

Gracias a la ayuda de Internet, ya hay varias empresas que han logrado disminuir su consumo de papel, sus empleados han sido entrenados para hacer todas sus transacciones sin moverse de sus escritorios, ahorrando cerca de 4.000 kilos de papel por semana en todo el mundo y cerca de 1000 millones de dólares en el 2001. Entre las operaciones más frecuentes que ya no requieren papel figuran: la confección de planillas de gastos, las compras y ventas, los pedidos de visas, las transferencias bancarias y los depósitos de sueldos. Esta práctica ayuda también a disminuir los niveles de corrupción, a aumentar la transparencia de los procesos, a hacer más con menos empleados y a asignar mejor los recursos económicos.

\section{LOS PROGRAMAS DE CONTA- BILIDAD ONLINE}

Permiten acceder a los datos desde cualquier lugar del mundo con conexión a Internet, éstas tienen las siguientes características:

- Son programas de bajo precio, por los que se paga una tarifa mensual

- Se accede con el propio navegador de Internet.

- El usuario dispone siempre de la última versión

- No necesita instalar el programa en su equipo

- Si surgen problemas los soluciona el fabricante.

También hay programas similares para realizar los impuestos, concretamente el programa de impuestos reside en el ordenador de un banco, lo que recuerda un poco la filosofia de las cajas de seguridad en las que los clientes guardan las joyas. 


\section{IA INFORMACIÓN ESTRATÉGICA}

Es necesario modificar los sistemas de información para dar cabida a la información del nivel estratégico que demanda la dirección de la empresa. Si seguimos el modelo de núcleo competitivo de Porter, los datos clave que se precisan en el nivel estratégico son relativos a:

- la competencia

- los clientes

- los proveedores

- las barreras a la entrada de nuevos competidores

- los productos sustitutos

Un sistema de información que no se limite a la informaciôn contable clásica, sino que de respuesta a estas necesidades de información debe informar sobre, por ejemplo: el tiempo medio que cuesta poner en marcha un nuevo producto en el mercado desde que se tiene la idea, el grado de calidad que trasmiten al consumidor nuestros productos frente a los de los competidores, indices $\mathrm{de}$ concentración del sector en el que opera nuestra empresa, etc.

Se deben diseñar sistemas de información por los que fluya el conocimiento de la organización y que favorezcan que los activos intangibles se usen de forma eficiente $y$ aumenten. Dos herramientas que posibilitan este uso son Intranet $y$ Groupware.

a) Intranet, consisten en aprovechar las herramientas de Internet para su utilización interna dentro de una empresa. Los empleados disponen de navegadores WWW para acceder a los datos internos de la em- presa -direcciones, datos, documentos internos de trabajo-, correo electrónico interno, utilidades para enviar ficheros, realizar videoconfe-rencias o reuniones electrónicas.

b) Groupware, aborda la gestión del conocimiento a través del trabajo en grupos utilizando las posibilidades de las tecnologias informáticas y de telecomu-nicaciones, combina las capacidades de gestión y consulta de documentos, con correo electrónico, agenda, navegador $\mathrm{Web}$, acceso a foros de noticias y videoconferencia.

\section{LOS SISTEMAS DE INFOR- MACIÓN INTEGRADOS, ENTER- PRISE RESOURCE PLANNING, O ERP}

Más que programas de ordenador son sistemas de información que integran aplicaciones informáticas para gestionar todos los departamentos y funciones de una empresa: contabilidad financiera $y$ analítica, finanzas, producción, mantenimiento, logística, recursos humanos, materiales, gestión de activos, compras y pagos, ventas y cobros, bancos y efectos, tesorería, cartera, gestión de proyectos, etc.

\section{LOS AUDITORES EN LA ERA DEL CONOCIMIENTO}

6.1Auditores Informáticos. La auditoría informática consiste en una revisión profunda $y$ detallada de todos los elementos de que dispone una empresa en el área de sistemas de información. Los conocimientos y 
experiencias que se exige a un auditor informático son relativos al diseño e implantación de sistemas de información, funcionamiento de un centro de cálculo y además ha de conocer los métodos administrativos y de gestión propios del sector en el que opera la empresa. Además ha de conocer las prestaciones del hardware instalado.

\subsection{La Auditoria Financiera sin} Papeles. La popularización de los ordenadores y el impacto de las tecnologías de la comunicación ha supuesto cambios radicales en los métodos y procedimientos utilizados por los auditores: "las tipicas hojas amarillas y los gruesos legajos de papeles, se han visto sustituidos por complejas hojas de cálculo y CD ROM conteniendo documentación escaneada. La utilización de libros contables ha dejado de ser una práctica habitual, dando lugar a accesos directos a ficheros contables de los que el auditor extrae sus pruebas y realiza sus análisis". Está claro que en el marco del comercio electrónico, los auditores deben modificar sus formas de trabajar. La proliferación de sistemas EDI conduce a la utilización cada vez más acentuada de técnicas de Auditoria Asistida por Ordenador (CAA).

Una iniciativa interesante de AICPA, el American Institute of Certified Public Accountants AICPA, es el Proyecto WebTrust que es un servicio proporcionado por Contadores Públicos especializados quienes determinan si un servidor Web sigue ciertos principios exigidos que permitan inspecciones de calidad sobre las transacciones electrónicas que realiza a través del World Wide Web después de haber cumplido con tres principios importantes:

- Divulgación de prácticas de negocios.

- Integridad de las transacciones. Mantiene controles efectivos que garantizan que las órdenes originadas por los consumidores a través de comercio electrónico se completan y se facturan según acordado por la institución y su cliente.

- Protección de la información. Mantiene controles efectivos para garantizar que la información privada del consumidor/cliente se protege de usos no relacionados con el negocio.

\subsection{Modelo de Dictamen de la Auditoria Financiera sin Papeles}

El dictamen debe obtener un certificado Web Trust, con la finalidad de garantizar dicho informe, que al más puro estilo de informe de auditoria comienza así:

"Hemos examinado la afirmación de la gerencia del Colegio de Contadores Públicos de Lima acerca de la divulgación de sus prácticas comerciales en su página interactiva para el comercio electrónico y la efectividad de sus controles para garantizar la integridad de las transacciones y la protección de la información utilizada para el comercio electrónico (en www.prscpa.org) durante el periodo del 1 de junio de 2002 al 31 de agosto de 2002"... “En nuestra opinión, durante el periodo del 1 de junio de 2002 al 31 de agosto de 2002 el Colegio, en todos los aspectos 
significativos: divulgó sus prácticas comerciales para el comercio electrónico y procesó las transacciones de acuerdo con las prácticas comerciales divulgadas, mantuvo controles efectivos que permiten afirmar, con certe$z a$ razonable, que las transacciones realizadas por los clientes a través del comercio electrónico fueron completadas y facturadas según lo acordado y mantuvo controles efectivos que le permiten afirmar, con certeza razonable, que la información privada de clientes obtenida como resultado del comercio electrónico fue protegida de usos no relacionados al comercio del Colegio". Esto significa que dicho servidor es un lugar seguro para navegar y realizar transacciones "on-line".

\section{CONCLUSIONES}

1. En esta Era Virtual y del Conocimiento las nuevas necesidades informativas de los usuarios deben ser satisfechas por los Contadores Públicos, tomando en cuenta las nuevas características que tiene la contabilidad para cumplir con esas necesidades de los usuarios, tales como:

- El Conocimiento-Educación continua

- La Velocidad-Eficiencia y eficacia.

- Globalización-Desmasificación, desintermediación, descentralización.

2. Los Intangibles que generan el Capital Intelectual en una empresa debe ser contabilizadas como un activo físico considerando para ello su identificación y valorización objetiva. Por lo que, deben ser expuesto en los estados financieros, con una nota aclaratoria que exponga la metodología adoptada en la determinación de su valor y estimación del periodo de vigencia del mismo.

3. El Contador Público debe prepararse para conocer a plenitud las tecnologías de la Información emergente en esta era del conocimiento ya que la implementación de la Tecnologia de la Información -TI no es simple, pero al ejecutarlo en los negocios debe sentarse en: Los Procesos, en su estructura organizacional y en la cultura organizacional.

4. En la Era del Conocimiento los investigadores de la Ciencia Contable deberán originar nuevos modelos y enfoques de Auditoria para ser aplicados en una Auditoría Continua, y sin papeles.

\section{BIBLIOGRAFÍA}

1. AICPA, The American Institute of Certified Public Accountants

2. IFAC, Guía Internacional de Educación $\mathrm{N}^{\circ} 11$, "Information Tecnology in the Accounting Curriculum".

3. IFAC, Federación Internacional de Contables, la sección de Tecnologías de Información, con documentos clave.

4. Boggs, S.M. (1999): “Accounting, the Digital Way", Journal of Accountancy, May 1999 Vol. 187 No. 5 
5. Cornellá, A. (1996): Información Digital para la Empresa, Ed. Marcombo, Barcelona.

6. Edvinsson, L. y Malone, M. (1997): "Intellectual Capital. Realizing your company's true value by finding its hidden brainpower", Harperbusiness

7. Elliot, R.K. (1992): “The Third Wave breaks on the Shores of Accounting", Accounting Horizons, June 1992, pag 61-85

8. Forkner, I. y McLeod, R (1982): Aplicaciones de la computadora a los sistemas administrativos, Ed Limusa, México 1982

9. King, A. M. (1999): Valuing Intangible Assets Through Appraisals, Strategic Finance, november 1999.

10. Monforte, M. (1995): Sistemas de Información para la Dirección, Ed. Pirámide, Madrid.

11. Wainman, D. (1995): "Voyage into the Digital Universe: Are CAs ready to meet the challenges posed by the new electronic frontier?", CA Magazine, August 1995. 